TOKYO J. MATH.

VOL. 27, No. 2, 2004

\title{
Stable Rank of Some Noncommutative Manifolds
}

\author{
Takahiro SUDO \\ University of the Ryukyus \\ (Communicated by K. Shinoda)
}

\begin{abstract}
In this paper we compute the stable rank and connected stable rank of some $C^{*}$-algebras regarded as noncommutative manifolds. Mainly, we consider the noncommutative spaces studied by Matsumoto and Tomiyama, their higher dimensional versions and the $C^{*}$-algebras of continuous fields of these noncommutative manifolds.
\end{abstract}

\section{Introduction}

The stable rank for $C^{*}$-algebras was introduced by M. A. Rieffel [Rf1], and the real rank was done by L. G. Brown and G. K. Pedersen [BP]. These ranks for commutative $C^{*}$-algebras correspond to the complex and real dimensions of topological spaces respectively. Also, the connected stable rank for $C^{*}$-algebras was introduced in [Rf1], and this rank for commutative $C^{*}$-algebras is related with vanishing of cohomotopy groups of spaces [Sh]. Moreover, the stable ranks are very important notions in the (non-stable) K-theory of $C^{*}$-algebras (cf. [Bl], [Rf1], [Rf2]). See References for some works about these ranks.

On the other hand, we have recently investigated the stable rank and connected stable rank of $C^{*}$-algebras of continuous fields [Sd]. In particular, we computed the stable ranks of the group $C^{*}$-algebras of the discrete Heisenberg groups regarded as the $C^{*}$-algebras of continuous fields of rotation algebras (cf. [AP]). In this paper, using the results of [Sd] ((F4) below) mainly we estimate the stable ranks of the noncommutative spaces studied by K. Matsumoto and J. Tomiyama ([Mt], [MT]), their higher dimensional versions and the $C^{*}$-algebras of continuous fields of these noncommutative manifolds. Also, our methods for calculating the stable ranks would be applicable to more other noncommutative manifolds.

Notation and facts. We first set up some notations and review some results of the stable ranks in the following.

Let $\mathfrak{A}$ be a $C^{*}$-algebra. We denote by $\operatorname{sr}(\mathfrak{A}), \operatorname{csr}(\mathfrak{A})$ the stable rank, connected stable rank respectively ([Rf1]). By definition, we have $\operatorname{sr}(\mathfrak{A}), \operatorname{csr}(\mathfrak{A}) \in\{1,2, \cdots, \infty\}$. If $\mathfrak{A}$ is nonunital, we define its ranks by those of the unitization $\mathfrak{A}^{+}$.

Received May 22, 2000; revised December 7, 2002

2000 Mathematics Subject Classification. Primary 46L05. Secondary 46L80, 19B10.

Research partially supported by Japan Society for the Promotion of Science 
(F1): For an exact sequence of $C^{*}$-algebras: $0 \rightarrow \mathfrak{I} \rightarrow \mathfrak{A} \rightarrow \mathfrak{A} / \mathfrak{I} \rightarrow 0$, we have

$$
\begin{aligned}
\operatorname{sr}(\mathfrak{I}) \vee \operatorname{sr}(\mathfrak{A} / \mathfrak{I}) \leq & \operatorname{sr}(\mathfrak{A}) \leq \operatorname{sr}(\mathfrak{I}) \vee \operatorname{sr}(\mathfrak{A} / \mathfrak{I}) \vee \operatorname{csr}(\mathfrak{A} / \mathfrak{I}), \\
& \operatorname{csr}(\mathfrak{A}) \leq \operatorname{csr}(\mathfrak{I}) \vee \operatorname{csr}(\mathfrak{A} / \mathfrak{I}),
\end{aligned}
$$

where $\vee$ is the maximum ([Rf1, Theorems 4.3, 4.4 and 4.11] and [Sh, Theorem 3.9]).

(F2): We denote by $C_{0}(X)$ the $C^{*}$-algebra of all continuous functions vanishing at infinity on a locally compact Hausdorff space $X$. If $X$ is compact, set $C_{0}(X)=C(X)$. By [Rf1, Proposition 1.7] and [Ns],

$$
\operatorname{sr}(C(X))=[\operatorname{dim} X / 2]+1, \quad \operatorname{csr}(C(X)) \leq[(\operatorname{dim} X+1) / 2]+1,
$$

where $\operatorname{dim} X$ is the covering dimension of $X$, and $[x]$ means the maximal integer $\leq x$. Set $\operatorname{dim}_{\mathbf{C}} X=[\operatorname{dim} X / 2]+1$. Note that $C_{0}(X)^{+} \cong C\left(X^{+}\right)$where $X^{+}$means the one-point compactification of $X$. Let $\beta X$ be the Stone-Čech compactification of $X$ (cf. $[\mathrm{Ng}]$ ).

(F3): For the $n \times n$ matrix algebra $M_{n}(\mathfrak{A})$ over a $C^{*}$-algebra $\mathfrak{A}$,

$$
\operatorname{sr}\left(M_{n}(\mathfrak{A})\right)=\{(\operatorname{sr}(\mathfrak{A})-1) / n\}+1, \quad \operatorname{csr}\left(M_{n}(\mathfrak{A})\right) \leq\{(\operatorname{csr}(\mathfrak{A})-1) / n\}+1,
$$

where $\{x\}$ means the least integer $\geq x([\operatorname{Rf} 1$, Theorem 6.1], [Rf2, Theorem 4.7]).

Let $X$ be a locally compact Hausdorff space and $\left\{\mathfrak{A}_{t}\right\}_{t \in X}$ a family of $C^{*}$-algebras $\mathfrak{A}_{t}$ indexed by $t \in X$. We denote by $\Gamma_{0}\left(X,\left\{\mathfrak{A}_{t}\right\}_{t \in X}, \mathfrak{F}\right)$ the $C^{*}$-algebra of a continuous field on $X$ with respect to a certain family $\mathfrak{F}$ of continuous operator fields vanishing at infinity on $X$ with some properties (cf. [F], [Dx], [Wl, Section 2]). If $X$ is compact, set $\Gamma_{0}\left(X,\left\{\mathfrak{A}_{t}\right\}_{t \in X}, \mathfrak{F}\right)=$ $\Gamma\left(X,\left\{\mathfrak{A}_{t}\right\}_{t \in X}, \mathfrak{F}\right)$. If $\mathfrak{A}_{t}=\mathfrak{A}$ for all $t \in X$, we put $\Gamma_{0}(X, \mathfrak{A}, \mathfrak{F})=\Gamma_{0}\left(X,\left\{\mathfrak{A}_{t}\right\}_{t \in X}, \mathfrak{F}\right)$. We omit $\mathfrak{F}$ in some cases in what follows. By [Sd], if $X$ is a locally compact, paracompact (or $\sigma$-compact or second countable) Hausdorff space, then

$$
\text { (F4): }\left\{\begin{array}{l}
\operatorname{sr}\left(\Gamma_{0}\left(X,\left\{\mathfrak{A}_{t}\right\}_{t \in X}, \mathfrak{F}\right)\right) \leq \sup _{t \in X} \operatorname{sr}\left(C_{0}\left(X, \mathfrak{A}_{t}\right)\right) \\
\operatorname{csr}\left(\Gamma_{0}\left(X,\left\{\mathfrak{A}_{t}\right\}_{t \in X}, \mathfrak{F}\right)\right) \leq \sup _{t \in X}\left(\operatorname{csr}\left(C_{0}\left(X, \mathfrak{A}_{t}\right)\right) \vee \operatorname{sr}\left(C_{0}\left(X, \mathfrak{A}_{t}\right)\right)\right)
\end{array}\right.
$$

where $C_{0}\left(X, \mathfrak{A}_{t}\right)$ is the $C^{*}$-algebra of all $\mathfrak{A}_{t}$-valued continuous functions on $X$ vanishing at infinity, which is isomorphic to the $C^{*}$-tensor product $C_{0}(X) \otimes \mathfrak{A}_{t}$ (cf. [Mp, Theorem 6.4.17]). Note that the assumption on $X$ is satisfied automatically in the cases below. On the other hand, by using [Rf1, Theorem 4.3] we have

$$
\sup _{t \in X} \operatorname{sr}\left(\mathfrak{A}_{t}\right) \leq \operatorname{sr}\left(\Gamma_{0}\left(X,\left\{\mathfrak{A}_{t}\right\}_{t \in X}, \mathfrak{F}\right)\right) .
$$

Let $\Gamma^{b}\left(X,\left\{\mathfrak{A}_{t}\right\}_{t \in X}, \mathfrak{F}\right)$ be the $C^{*}$-algebra of a bounded continuous field on $X$ with respect to a family $\mathfrak{F}$ of bounded continuous operator fields on $X$, and $C^{b}(X)$ the $C^{*}$-algebra of all bounded continuous functions on $X$. The formulas (F4), (F5) hold when replacing $\Gamma_{0}(\cdot)$, $C_{0}\left(X, \mathfrak{A}_{t}\right)$ with $\Gamma^{b}(\cdot), C^{b}(X) \otimes \mathfrak{A}_{t}$ respectively if $X$ is $\sigma$-compact and $\mathfrak{F}$ contains the unit field when all $\mathfrak{A}_{t}$ are unital.

Denote by $M(\mathfrak{I})$ the multiplier of a $C^{*}$-algebra $\mathfrak{I}$ (cf. [APT], [Wo]). 


\section{Noncommutative manifolds}

1.1. Noncommutative tori. Let $\mathbf{T}_{\theta}^{2}$ be the rotation algebra by the angle $2 \pi \theta$ defined by the $C^{*}$-crossed product $C(\mathbf{T}) \rtimes_{\theta} \mathbf{Z}$ with the action of $\mathbf{Z}$ on the torus $\mathbf{T}$ by the multiplication $e^{2 \pi i \theta n} z$ for $n \in \mathbf{Z}, z \in \mathbf{T}$ and $\theta \in[0,1]$. Then it is known that

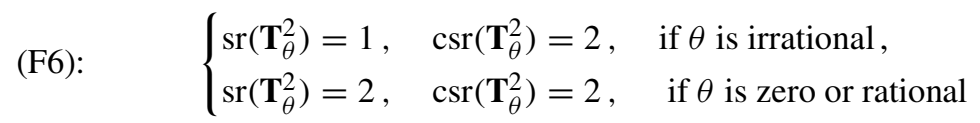

(cf. [AP], [EE], [DNNP], [Sh, p. 381], [Eh, Theorem 2.2], [Rf1, Corollary 4.10], [Sd]).

A noncommutative $n$-torus is the $C^{*}$-algebra of generated by $n$-unitaries $U_{j}$ with the commutation relation $U_{j} U_{k}=e^{2 \pi i \theta_{j, k}} U_{k} U_{j}$ with $\theta_{j, k} \in \mathbf{R}$ for $1 \leq j, k \leq n$. In particular, $\mathbf{T}_{\theta}^{2}$ is a noncommutative 2-torus. The structure of some (simple) noncommutative tori are studied by $[\mathrm{EL} 1,2],[\mathrm{Ln}]$ to get their inductive limit decompositions as AT-algebras, i.e. inductive limits of matrix algebras over $C(\mathbf{T})$, which are useful to calculate their ranks. On the other hand, by [BKR] simple noncommutative tori have the same ranks with $\mathbf{T}_{\theta}^{2}$ for $\theta$ irrational.

1.2. Noncommutative solid torus. The noncommutative solid torus defined by Matsumoto [Mt] means the crossed product $\left(D^{2} \times \mathbf{T}\right)_{\theta}=C\left(D^{2}\right) \rtimes_{\theta} \mathbf{Z}$ with the action of $\mathbf{Z}$ on the 2-dimensional unit closed disk $D^{2}$ by the rotation by the angle $2 \pi \theta$ with $0<\theta<1$. Then by [MT, Proposition 6.4], $\left(D^{2} \times \mathbf{T}\right)_{\theta}$ is regarded as the $C^{*}$-algebra of a continuous field on the interval $[0,1]$ with fibers $\mathfrak{A}_{t}$ for $t \in[0,1]$ given by

$$
\mathfrak{A}_{0}=C(\mathbf{T}), \quad \mathfrak{A}_{t}=\mathbf{T}_{\theta}^{2} \quad \text { for } t \neq 0 .
$$

Then by $(\mathrm{F} 4)$, we obtain $\operatorname{sr}\left(\left(D^{2} \times \mathbf{T}\right)_{\theta}\right) \leq \operatorname{sr}(C([0,1] \times \mathbf{T})) \vee \operatorname{sr}\left(C\left([0,1], \mathbf{T}_{\theta}^{2}\right)\right)$ and $\operatorname{csr}\left(\left(D^{2} \times \mathbf{T}\right)_{\theta}\right) \leq \operatorname{csr}(C([0,1] \times \mathbf{T})) \vee \operatorname{csr}\left(C\left([0,1], \mathbf{T}_{\theta}^{2}\right)\right) \vee \operatorname{sr}(C([0,1] \times \mathbf{T})) \vee \operatorname{sr}(C([0,1]$, $\left.\mathbf{T}_{\theta}^{2}\right)$ ). By the same way as (F6) (cf. [Sd]) and using [Rf1, Theorem 5.1], (F3), [NOP, Proposition 5.2] and [Eh, Corollary 2.12], we obtain

$$
\operatorname{sr}\left(C\left([0,1], \mathbf{T}_{\theta}^{2}\right)\right)=2, \quad \operatorname{csr}\left(C\left([0,1], \mathbf{T}_{\theta}^{2}\right)\right)=2
$$

for any nonzero $\theta$. Note that $\mathbf{T}_{\theta}^{2}$ for $\theta$ irrational is an AT-algebra and $\mathbf{T}_{\theta}^{2}$ for $\theta$ rational is homogeneous, and the $K_{1}$-group of $C\left([0,1], \mathbf{T}_{\theta}^{2}\right)$ is nontrivial. Hence $\operatorname{sr}\left(\left(D^{2} \times \mathbf{T}\right)_{\theta}\right) \leq 2$ and $\operatorname{csr}\left(\left(D^{2} \times \mathbf{T}\right)_{\theta}\right) \leq 2$. On the other hand, we have the following exact sequence:

$$
0 \rightarrow \mathfrak{I} \rightarrow\left(D^{2} \times \mathbf{T}\right)_{\theta} \rightarrow C(\mathbf{T}) \rightarrow 0
$$

where $\mathfrak{I}=C_{0}\left((0,1], \mathbf{T}_{\theta}^{2}\right)$. Then by (F5) and [Eh, Theorem 2.2] we have

$$
\operatorname{sr}\left(\left(D^{2} \times \mathbf{T}\right)_{\theta}\right) \geq \operatorname{sr}(\mathfrak{I})=2, \quad \operatorname{csr}\left(\left(D^{2} \times \mathbf{T}\right)_{\theta}\right) \geq 2 .
$$

Summing up we obtain

THEOREM 1.1. Let $\left(D^{2} \times \mathbf{T}\right)_{\theta}$ be the noncommutative solid torus. Then we have

$$
\operatorname{sr}\left(\left(D^{2} \times \mathbf{T}\right)_{\theta}\right)=2, \quad \operatorname{csr}\left(\left(D^{2} \times \mathbf{T}\right)_{\theta}\right)=2 .
$$


REMARK. If $\theta=0$, then $\left(D^{2} \times \mathbf{T}\right)_{0} \cong C\left(D^{2} \times \mathbf{T}\right)$. By (F2), [Ns, Corollary 2.9] and [Sh, p. 381], we have $\operatorname{sr}\left(\left(D^{2} \times \mathbf{T}\right)_{0}\right)=2$ and $\operatorname{csr}\left(\left(D^{2} \times \mathbf{T}\right)_{0}\right)=\operatorname{csr}(C(\mathbf{T}))=2$.

1.3. Higher dimensional case of $\left(D^{2} \times \mathbf{T}\right)_{\theta}$. Let $\left(\Pi^{n} D^{2} \times \mathbf{T}\right)_{\Theta}=C\left(\Pi^{n} D^{2}\right) \rtimes_{\Theta} \mathbf{Z}$ be the higher dimensional, noncommutative solid torus with the action of $\mathbf{Z}$ on the $n$-direct product $\Pi^{n} D^{2}$ of $D^{2}$ by the multi-rotation by $\left(e^{2 \pi i \theta_{j} t}\right)_{j=1}^{n}$ with $t \in \mathbf{Z}, \Theta=\left(\theta_{j}\right)_{j=1}^{n}, 0<$ $\theta_{j}<1$. Then $\left(\Pi^{n} D^{2} \times \mathbf{T}\right)_{\theta}$ is regarded as the $C^{*}$-algebra of a continuous field on $[0,1]^{n}$ with fibers $\mathfrak{A}_{t}$ for $t \in[0,1]^{n}$ given by

$$
\mathfrak{A}_{t}= \begin{cases}C(\mathbf{T}) & t=0_{n}=(0, \cdots, 0), \\ \mathbf{T}_{\Theta\left(j_{1}, \cdots, j_{k}\right)}^{k+1}=\left(t_{1}, \cdots, t_{n}\right), t_{j_{s}} \neq 0(1 \leq s \leq k), \\ \mathbf{T}_{\Theta}^{n+1} & t=\left(t_{1}, \cdots, t_{n}\right), t_{j} \neq 0(1 \leq j \leq n)\end{cases}
$$

where $\mathbf{T}_{\Theta\left(j_{1}, \cdots, j_{k}\right)}^{k+1}=C\left(\mathbf{T}^{k}\right) \rtimes_{\Theta\left(j_{1}, \cdots, j_{k}\right)} \mathbf{Z}$ and $\mathbf{T}_{\Theta}^{n+1}=C\left(\mathbf{T}^{n}\right) \rtimes_{\Theta} \mathbf{Z}$ are noncommutative tori, and $\Theta\left(j_{1}, \cdots, j_{k}\right)=\left(\theta_{j_{s}}\right)_{s=1}^{k}$. Then by (F4), we obtain

$$
\begin{aligned}
\operatorname{sr}\left(\left(\Pi^{n} D^{2} \times \mathbf{T}\right)_{\Theta}\right) \leq & \operatorname{sr}\left(C\left([0,1]^{n} \times \mathbf{T}\right)\right) \vee \max _{1 \leq k \leq n} \operatorname{sr}\left(C\left([0,1]^{n}, \mathbf{T}_{\Theta\left(j_{1}, \cdots, j_{k}\right)}^{k+1}\right)\right) \\
\operatorname{csr}\left(\left(\Pi^{n} D^{2} \times \mathbf{T}\right)_{\Theta}\right) \leq & \operatorname{csr}\left(C\left([0,1]^{n} \times \mathbf{T}\right)\right) \vee \max _{1 \leq k \leq n} \operatorname{csr}\left(C\left([0,1]^{n}, \mathbf{T}_{\Theta\left(j_{1}, \cdots, j_{k}\right)}^{k+1}\right)\right) \\
& \vee \operatorname{sr}\left(C\left([0,1]^{n} \times \mathbf{T}\right)\right) \vee \max _{1 \leq k \leq n} \operatorname{sr}\left(C\left([0,1]^{n}, \mathbf{T}_{\Theta\left(j_{1}, \cdots, j_{k}\right)}^{k+1}\right)\right) .
\end{aligned}
$$

By the same way as the case $n=1$ (cf. [Sd]), if all $\theta_{j}$ are irrational, or if some of $\theta_{j}$ are rational and their periods are large enough, then we obtain

$$
\left\{\begin{array}{l}
\operatorname{sr}\left(C\left([0,1]^{n}, \mathbf{T}_{\Theta\left(j_{1}, \cdots, j_{k}\right)}^{k+1}\right)\right)=2, \\
\operatorname{csr}\left(C\left([0,1]^{n}, \mathbf{T}_{\Theta\left(j_{1}, \cdots, j_{k}\right)}^{k+1}\right)\right)=\operatorname{csr}\left(\mathbf{T}_{\Theta\left(j_{1}, \cdots, j_{k}\right)}^{k+1}\right)=2
\end{array}\right.
$$

for any $1 \leq k \leq n$ (cf. [Eh, Corollary 2.12]). However, in general we have

$$
\left\{\begin{array}{l}
\operatorname{sr}\left(C\left([0,1]^{n}, \mathbf{T}_{\Theta\left(j_{1}, \cdots, j_{k}\right)}^{k+1}\right)\right) \leq\{[(n+k) / 2] / q\}+1 \\
\operatorname{csr}\left(C\left([0,1]^{n}, \mathbf{T}_{\Theta\left(j_{1}, \cdots, j_{k}\right)}^{k+1}\right)\right)=\operatorname{csr}\left(\mathbf{T}_{\Theta\left(j_{1}, \cdots, j_{k}\right)}^{k+1}\right) \leq\{[(k+1) / 2] / q\}+1
\end{array}\right.
$$

where $q$ is the period of orbits by the action on $\mathbf{T}^{k}$ by $\Theta\left(j_{1}, \cdots, j_{k}\right)$ rational. Note that $\mathbf{T}_{\Theta\left(j_{1}, \cdots, j_{k}\right)}^{k+1}$ is homogeneous when all $\theta_{j_{s}}(1 \leq s \leq k)$ are rational and use (F2) and (F3) for its rank estimates. On the other hand, we have the following exact sequence:

$$
0 \rightarrow \mathfrak{I} \rightarrow\left(\Pi^{n} D^{2} \times \mathbf{T}\right)_{\Theta} \rightarrow C(\mathbf{T}) \rightarrow 0
$$

where $\mathfrak{I}=\Gamma_{0}\left(X,\left\{\mathfrak{A}_{t}\right\}_{t \in X}\right)$ with $X=[0,1]^{n} \backslash\left\{0_{n}\right\}$. Then we have

$$
\operatorname{sr}\left(\left(\Pi^{n} D^{2} \times \mathbf{T}\right)_{\Theta}\right) \geq \operatorname{sr}\left(\left(D^{2} \times \mathbf{T}\right)_{\theta_{j}}\right)=2, \quad \operatorname{csr}\left(\left(\Pi^{2} D^{2} \times \mathbf{T}\right)_{\Theta}\right) \geq 2
$$


where the first inequality follows from that $\left(D^{2} \times \mathbf{T}\right)_{\theta_{j}}$ is a quotient of $\left(\Pi^{n} D^{2} \times \mathbf{T}\right)_{\Theta}$, and the second one is obtained by [Eh, Theorem 2.2]. By (F1) and (F4), we also get

$$
\begin{aligned}
\operatorname{csr}\left(\left(\Pi^{n} D^{2} \times \mathbf{T}\right)_{\Theta}\right) & \leq \operatorname{csr}(\mathfrak{I}) \vee \operatorname{csr}(C(\mathbf{T})) \\
& \leq \sup _{t \in X}\left(\operatorname{csr}\left(C_{0}\left(X, \mathfrak{A}_{t}\right)\right) \vee \operatorname{sr}\left(C_{0}\left(X, \mathfrak{A}_{t}\right)\right)\right) \vee 2 .
\end{aligned}
$$

Summing up we obtain

THEOREM 1.2. Let $\left(\Pi^{n} D^{2} \times \mathbf{T}\right)_{\Theta}$ be the higher dimensional, noncommutative solid torus. Then we have

$$
\left\{\begin{array}{l}
2 \leq \operatorname{sr}\left(\left(\Pi^{n} D^{2} \times \mathbf{T}\right)_{\Theta}\right) \leq\{n / q\}+1, \\
2 \leq \operatorname{csr}\left(\left(\Pi^{n} D^{2} \times \mathbf{T}\right)_{\Theta}\right) \leq\{n / q\}+1,
\end{array}\right.
$$

where $q$ is the least period of orbits by the rational action by $\Theta$ non-irrational. If all $\theta_{j}$ are irrational, or if some of $\theta_{j}$ are rational and $q$ is large enough, then

$$
\operatorname{sr}\left(\left(\Pi^{n} D^{2} \times \mathbf{T}\right)_{\Theta}\right)=2, \quad \operatorname{csr}\left(\left(\Pi^{n} D^{2} \times \mathbf{T}\right)_{\Theta}\right)=2 .
$$

Remark. If $\Theta=0=(0)_{j=1}^{n}$, then $\left(\Pi^{n} D^{2} \times \mathbf{T}\right)_{0} \cong C\left(\Pi^{n} D^{2} \times \mathbf{T}\right)$. By (F2), [Ns, Corollary 2.9], [Eh, Corollary 2.12] and [Sh, p. 381], we get

$$
\operatorname{sr}\left(\left(\Pi^{n} D^{2} \times \mathbf{T}\right)_{0}\right)=n+1, \quad \operatorname{csr}\left(\left(\Pi^{n} D^{2} \times \mathbf{T}\right)_{0}\right)=\operatorname{csr}(C(\mathbf{T}))=2 .
$$

More generally, we can consider the following crossed products by the same way:

$$
\left(\left(\Pi^{k} D^{2} \times \Pi^{n-k} \mathbf{T}\right) \times \mathbf{T}\right)_{\Theta}=C\left(\Pi^{k} D^{2} \times \Pi^{n-k} \mathbf{T}\right) \rtimes_{\Theta} \mathbf{Z} .
$$

Moreover, it would be interesting to consider the crossed products $C\left(D^{n}\right) \rtimes_{\alpha} \mathbf{Z}$ with $\alpha$ suitable actions on the $n$-dimensional unit disk $D^{n}$ as a higher dimensional case.

1.4. Noncommutative lens spaces. The noncommutative lens spaces are defined and studied in detail by Matsumoto and Tomiyama [MT]. For coprime integers $p, q$, we denote by $L_{\theta}(p, q)$ the noncommutative lens space of type $(p, q)$ defined by the pull back satisfying the following diagram:

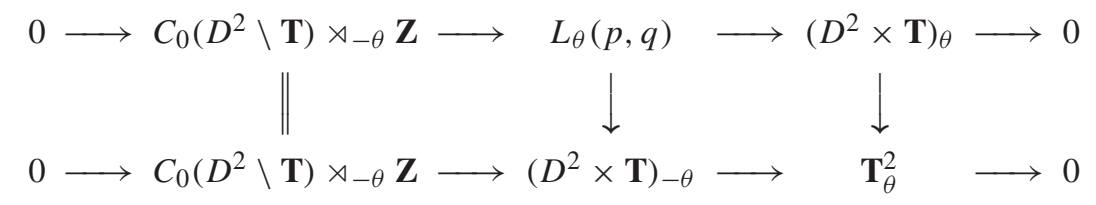

where $C_{0}\left(D^{2} \backslash \mathbf{T}\right) \rtimes_{-\theta} \mathbf{Z}$ means the crossed product by $\mathbf{Z}$ by the rotation by $-\theta$. Moreover, by [MT, Theorem 6.8], $L_{\theta}(p, q)$ is regarded as the $C^{*}$-algebra of a continuous field on the interval $[-1,1]$ with fibers $\mathfrak{A}_{t}$ given by

$$
\mathfrak{A}_{-1}=C^{*}\left(V^{q} U^{p}\right), \quad \mathfrak{A}_{t}=\mathbf{T}_{\theta}^{2} \quad \text { for }-1<t<1, \quad \mathfrak{A}_{1}=C^{*}(V)
$$


where $C^{*}\left(V^{q} U^{p}\right), C^{*}(V)$ are $C^{*}$-algebras generated by $V^{q} U^{p}, V$ respectively, and $U, V$ are canonical unitary generators of $\mathbf{T}_{\theta}^{2}$ with the relation $V U=e^{2 \pi i \theta} U V$. By (F4),

$$
\operatorname{sr}\left(L_{\theta}(p, q)\right) \leq 2, \quad \operatorname{csr}\left(L_{\theta}(p, q)\right) \leq 2 .
$$

By Theorem 1.1 and $(\mathrm{F} 1)$, we have $\operatorname{sr}\left(L_{\theta}(p, q)\right) \geq 2$. By [Eh, Corollary 1.6], we obtain $\operatorname{csr}\left(L_{\theta}(p, q)\right) \geq 2$ since the $K_{1}$-group of $L_{\theta}(p, q)$ is nontrivial [MT, Proposition 2.2]. Combining [NOP, Proposition 1.6] with Theorem 1.1, we also obtain

$$
\operatorname{sr}\left(L_{\theta}(p, q)\right) \leq \operatorname{sr}\left(\left(D^{2} \times \mathbf{T}\right)_{\theta}\right) \vee \operatorname{sr}\left(\left(D^{2} \times \mathbf{T}\right)_{-\theta}\right) \leq 2 .
$$

Consequently, we have

THEOREM 1.3. Let $L_{\theta}(p, q)$ be the noncommutative lens space of type $(p, q)$. Then

$$
\operatorname{sr}\left(L_{\theta}(p, q)\right)=2, \quad \operatorname{csr}\left(L_{\theta}(p, q)\right)=2 .
$$

REMARK. If $\theta=0$, then $L_{0}(p, q) \cong C(L(p, q))$, where $L(p, q)$ is the lens space of type $(p, q)$ obtained by the quotient space of the 3-dimensional sphere $S^{3}$ by the action $\tau$ of a cyclic group defined by $\tau(z, w)=\left(e^{2 \pi i / p} z, e^{2 q \pi i / p} w\right)$ for $(z, w) \in S^{3} \subset \mathbf{C}^{2}$. In particular, $L(1,0)=S^{3}$. By (F2), [Sh, p. 381] and [Eh, Theorem 1.1] we have

$$
\begin{aligned}
& \operatorname{sr}(C(L(p, q)))=2=\operatorname{sr}\left(C\left(S^{3}\right)\right), \\
& \operatorname{csr}(C(L(p, q))) \leq \operatorname{csr}\left(C\left(S^{3}\right)\right) \vee \operatorname{sr}\left(C\left(S^{3}\right)\right)=3 .
\end{aligned}
$$

1.5. Higher dimensional case of $L_{\theta}(p, q)$. We define the higher dimensional, noncommutative lens space $L_{\Theta}^{n}$ to be the pull back satisfying the following diagram:

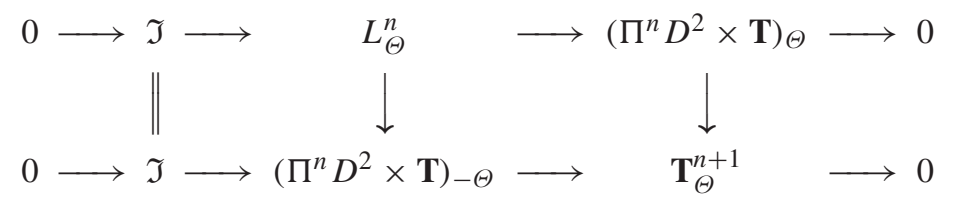

where $\mathfrak{I}=C_{0}\left(\Pi^{n} D^{2} \backslash \mathbf{T}^{n}\right) \rtimes_{-\Theta} \mathbf{Z}$ with the action by the multi-rotation by $-\Theta$. By Theorem 1.3 and (F2), we have $\operatorname{sr}\left(L_{\Theta}^{n}\right) \geq 2$. By [Eh, Corollary 1.6], we obtain $\operatorname{csr}\left(L_{\Theta}^{n}\right) \geq 2$ since the $K_{1}$-group of $L_{\Theta}^{n}$ is nontrivial by the similar calculation as [MT, Proposition 2.2]. By [NOP, Proposition 1.6] and (F1), we obtain

$$
\left\{\begin{array}{l}
\operatorname{sr}\left(L_{\theta}^{n}\right) \leq \operatorname{sr}\left(\left(\Pi^{n} D^{2} \times \mathbf{T}\right)_{\Theta}\right) \vee \operatorname{sr}\left(\left(\Pi^{n} D^{2} \times \mathbf{T}\right)_{-\Theta}\right), \\
\operatorname{csr}\left(L_{\theta}^{n}\right) \leq \operatorname{csr}(\mathfrak{I}) \vee \operatorname{csr}\left(\left(\Pi^{n} D^{2} \times \mathbf{T}\right)_{\Theta}\right)
\end{array}\right.
$$

Since $\mathfrak{I}$ is regarded as the $C^{*}$-algebra of a continuous field on $\Pi^{n} D^{2} \backslash \mathbf{T}^{n}$, the $\operatorname{rank} \operatorname{csr}(\mathfrak{I})$ is estimated by the same way as Theorem 1.2. By Theorem 1.2, we conclude

THEOREM 1.4. Let $L_{\Theta}^{n}$ be the higher dimensional, noncommutative lens space. Then

$$
2 \leq \operatorname{sr}\left(L_{\Theta}^{n}\right) \leq\{n / q\}+1, \quad 2 \leq \operatorname{csr}\left(L_{\Theta}^{n}\right) \leq\{n / q\}+1,
$$


where $q$ is the least period of orbits by the rational action by $\Theta$ non-irrational. If all $\theta_{j}$ are irrational, or if some of $\theta_{j}$ are rational and their periods are large enough, then

$$
\operatorname{sr}\left(L_{\Theta}^{n}\right)=2, \quad \operatorname{csr}\left(L_{\Theta}^{n}\right)=2 .
$$

REMARK. If $\Theta=0$, then $L_{0}^{n} \cong C\left(Y_{n}\right)$ where $Y_{n}$ is a certain space obtained by suitably attaching $\Pi^{n} D^{2} \times \mathbf{T}$ with $\left(\Pi^{n} D^{2} \backslash \mathbf{T}^{n}\right) \times \mathbf{T}$ (on $\mathbf{T}^{n+1}$ ). Hence, by (F2), [Sh, p. 381] and [Eh, Theorem 1.1] we have

$$
\operatorname{sr}\left(C\left(L_{0}^{n}\right)\right)=n+1=\operatorname{sr}\left(C\left(S^{2 n+1}\right)\right), \quad \operatorname{csr}\left(C\left(L_{0}^{n}\right)\right) \leq n+2=\operatorname{csr}\left(C\left(S^{2 n+1}\right)\right) .
$$

On the other hand, in definition of $L_{\Theta}^{n}$ we may take various $\left(\left(\Pi^{k} D^{2} \times \Pi^{n-k} \mathbf{T}\right) \times \mathbf{T}\right)_{\Theta}$ and $\left(\left(\Pi^{k^{\prime}} D^{2} \times \Pi^{n-k^{\prime}} \mathbf{T}\right) \times \mathbf{T}\right)_{-\Theta}$ as in the remark of Theorem 1.2. It would be preferable to take two crossed products of the form $C\left(D^{2 n}\right) \rtimes_{\alpha} \mathbf{Z}$ as definition of $L_{\Theta}^{n}$.

\section{Continuous fields of noncommutative manifolds}

In this section, we consider the $C^{*}$-algebras of continuous fields with fibers given by the noncommutative manifolds in the previous section. We identify elements $e^{2 \pi i \theta}$ of $\mathbf{T}$ with $\theta \in[0,1](\bmod 1)$. By the same way with Theorem 1.1 we have

THEOREM 2.1. Let $\mathbf{T}_{\theta}^{2}$ be the rotation algebra for $\theta \in[0,1]$. Then we have

$$
\left\{\begin{array}{l}
\operatorname{sr}\left(\Gamma\left(\mathbf{T},\left\{\otimes^{n} \mathbf{T}_{\theta}^{2}\right\}_{\theta \in \mathbf{T}}, \mathfrak{F}\right)\right)=n+1=\operatorname{sr}\left(C\left(\Pi^{n} \mathbf{T}^{2}\right)\right) \\
\operatorname{csr}\left(\Gamma\left(\mathbf{T},\left\{\otimes^{n} \mathbf{T}_{\theta}^{2}\right\}_{\theta \in \mathbf{T}}, \mathfrak{F}\right)\right) \leq n+1=\operatorname{csr}\left(C\left(\Pi^{n} \mathbf{T}^{2}\right)\right)
\end{array}\right.
$$

where $\mathfrak{F}$ contains the unit field. Moreover, taking the restriction to $\mathbf{T} \backslash\{1\}$, we get

$$
\left\{\begin{array}{l}
\operatorname{sr}\left(\Gamma_{0}\left(\mathbf{T} \backslash\{1\},\left\{\otimes^{n} \mathbf{T}_{\theta}^{2}\right\}_{\theta \in \mathbf{T} \backslash\{1\}}, \mathfrak{F}\right)\right)=2, \\
\operatorname{csr}\left(\Gamma_{0}\left(\mathbf{T} \backslash\{1\},\left\{\otimes^{n} \mathbf{T}_{\theta}^{2}\right\}_{\theta \in \mathbf{T} \backslash\{1\}}, \mathfrak{F}\right)\right)=2 .
\end{array}\right.
$$

Proof. Note that the following diagram is commutative:

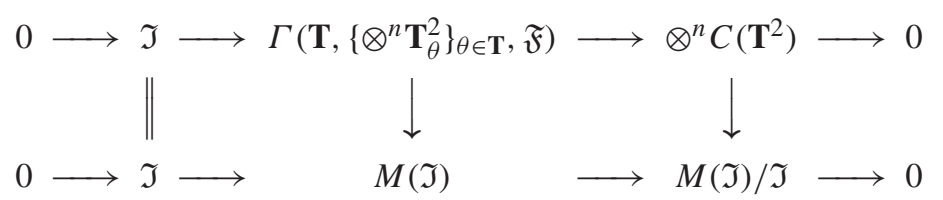

where $\mathfrak{I}=\Gamma_{0}\left(\mathbf{T} \backslash\{1\},\left\{\otimes^{n} \mathbf{T}_{\theta}^{2}\right\}_{\theta \in \mathbf{T} \backslash\{1\}}\right)$ and $M(\mathfrak{I}) \cong \Gamma^{b}\left(\mathbf{T} \backslash\{1\},\left\{\otimes^{n} \mathbf{T}_{\theta}^{2}\right\}_{\theta \in \mathbf{T} \backslash\{1\}}\right)$ since the unit field is continuous with respect to $\mathfrak{F}$ ([APT, p. 286]). Use [Sh, p. 381].

REMARK. The group $C^{*}$-algebra of the discrete Heisenberg group is a special case of $\Gamma\left(\mathbf{T},\left\{\mathbf{T}_{\theta}^{2}\right\}_{\theta \in \mathbf{T}}, \mathfrak{F}\right)(\mathrm{cf} .[\mathrm{AP}],[\mathrm{Sd}])$. 
THEOREM 2.2. Let $\left\{\mathfrak{A}_{\theta}\right\}_{\theta \in \mathbf{T}}=\left\{\otimes^{n}\left(D^{2} \times \mathbf{T}\right)_{\theta}\right\}_{\theta \in \mathbf{T}}$ or $\left\{\otimes^{n} L_{\theta}(p, q)\right\}_{\theta \in \mathbf{T}}$. Then we have

$$
\operatorname{sr}\left(\Gamma\left(\mathbf{T},\left\{\mathfrak{A}_{\theta}\right\}_{\theta \in \mathbf{T}}, \mathfrak{F}\right)\right)=[3 n / 2]+1=\operatorname{sr}(C(X))=\operatorname{sr}(C(Y)),
$$

where $\mathfrak{F}$ contains the unit field, and

$$
\begin{aligned}
& \operatorname{csr}\left(\Gamma\left(\mathbf{T},\left\{\otimes^{n}\left(D^{2} \times \mathbf{T}\right)_{\theta}\right\}_{\theta \in \mathbf{T}}, \mathfrak{F}\right)\right) \leq[(n+1) / 2]+1=\operatorname{csr}(C(X)), \\
& \left.\operatorname{csr}\left(\Gamma\left(\mathbf{T},\left\{\otimes^{n} L_{\theta}(p, q)\right\}_{\theta}\right\}_{\theta \in \mathbf{T}}, \mathfrak{F}\right)\right) \leq[(3 n+1) / 2]+1
\end{aligned}
$$

where $X=\Pi^{n}\left(D^{2} \times \mathbf{T}\right)$ and $Y=\Pi^{n} L(p, q)$. Moreover, we have

$$
\operatorname{sr}\left(\Gamma_{0}\left(\mathbf{T} \backslash\{1\},\left\{\mathfrak{A}_{\theta}\right\}_{\theta \in \mathbf{T} \backslash\{1\}}\right)\right)=2, \quad \operatorname{csr}\left(\Gamma_{0}\left(\mathbf{T} \backslash\{1\},\left\{\mathfrak{A}_{\theta}\right\}_{\theta \in \mathbf{T} \backslash\{1\}}\right)\right)=2 .
$$

PROOF. Note that the following diagram is commutative:

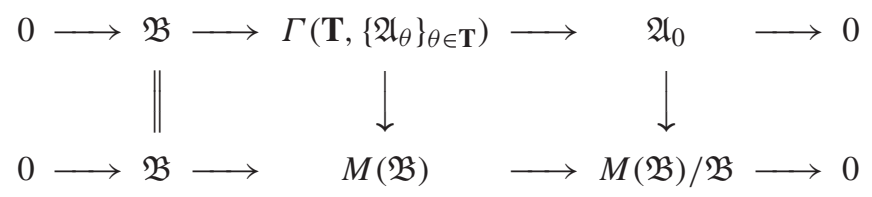

where $\mathfrak{B}=\Gamma_{0}\left(\mathbf{T} \backslash\{1\},\left\{\mathfrak{A}_{\theta}\right\}_{\theta \in \mathbf{T} \backslash\{1\}}\right)$ and $M(\mathfrak{B}) \cong \Gamma^{b}\left(\mathbf{T} \backslash\{1\},\left\{\mathfrak{A}_{\theta}\right\}_{\theta \in \mathbf{T} \backslash\{1\}}\right)$. Therefore, by [NOP, Proposition 1.6], (F1) and (F4) we get

$$
\begin{aligned}
\operatorname{sr}\left(\mathfrak{A}_{0}\right) & \leq \operatorname{sr}\left(\Gamma\left(\mathbf{T},\left\{\mathfrak{A}_{\theta}\right\}_{\theta \in \mathbf{T}}\right)\right) \leq \operatorname{sr}\left(\mathfrak{A}_{0}\right) \vee \operatorname{sr}(M(\mathfrak{B})) \\
& \leq([3 n / 2]+1) \vee \sup _{\theta \in \mathbf{T} \backslash\{1\}} \operatorname{sr}\left(C^{b}(\mathbf{T} \backslash\{1\}) \otimes \mathfrak{A}_{\theta}\right), \\
\operatorname{csr}\left(\mathfrak{A}_{0}\right) & \leq \operatorname{csr}\left(\Gamma\left(\mathbf{T},\left\{\mathfrak{A}_{\theta}\right\}_{\theta \in \mathbf{T}}\right)\right) \leq \operatorname{csr}\left(\mathfrak{A}_{0}\right) \vee \operatorname{csr}(\mathfrak{B}) \\
& \leq \operatorname{csr}\left(\mathfrak{A}_{0}\right) \vee \sup _{\theta \in \mathbf{T} \backslash\{1\}}\left(\operatorname{csr}\left(C_{0}(\mathbf{T} \backslash\{1\}) \otimes \mathfrak{A}_{\theta}\right) \vee \operatorname{sr}\left(C_{0}(\mathbf{T} \backslash\{1\}) \otimes \mathfrak{A}_{\theta}\right)\right) .
\end{aligned}
$$

Note that $\operatorname{csr}\left(C\left(\Pi^{n}\left(D^{2} \times \mathbf{T}\right)\right)\right)=\operatorname{csr}\left(C\left(\mathbf{T}^{n}\right)\right)=[(n+1) / 2]+1$. On the other hand, we have $\operatorname{csr}\left(C\left(\Pi^{n} L(p, q)\right)\right) \leq \operatorname{csr}\left(C\left(\Pi^{n}\left(D^{2} \times \mathbf{T}\right)\right)\right) \vee \operatorname{sr}\left(C\left(\Pi^{n}\left(D^{2} \times \mathbf{T}\right)\right)\right)=[(3 n+1) / 2]+1$ by [Eh, Theorem 1.1]. Also, note that $C^{b}(\mathbf{T} \backslash\{1\}) \cong C(\beta(\mathbf{T} \backslash\{1\}))$ and $\operatorname{dim} \beta(\mathbf{T} \backslash\{1\})=$ $\operatorname{dim} \mathbf{T} \backslash\{1\}=1$.

Now assume that $\mathfrak{A}_{\theta}=\otimes^{n}\left(D^{2} \times \mathbf{T}\right)_{\theta}$. The case for $\otimes^{n} L_{\theta}(p, q)$ is treated similarly. Then $C(\mathbf{T}) \otimes \mathfrak{A}_{\theta}$ is regarded as the $C^{*}$-algebra of a continuous field on $[0,1]$ with the following fibers:

$$
\mathfrak{B}_{\theta, t}=C\left(\mathbf{T}^{n+1}\right) \quad \text { at } t=0, \quad \mathfrak{B}_{\theta, t}=C\left(\mathbf{T}, \otimes^{n} \mathbf{T}_{\theta}^{2}\right) \quad \text { for } t \neq 0 .
$$

Note that the following diagram is commutative:

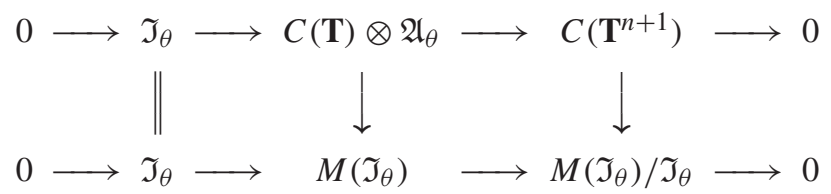


where $\mathfrak{I}_{\theta}=C_{0}\left(\mathbf{T} \times(0,1], \otimes^{n} \mathbf{T}_{\theta}^{2}\right)$. Therefore, we obtain for $\theta \in \mathbf{T} \backslash\{1\}$,

$$
\begin{aligned}
& \operatorname{sr}\left(C(\mathbf{T}) \otimes \mathfrak{A}_{\theta}\right) \leq \operatorname{sr}\left(C\left(\mathbf{T}^{n+1}\right)\right) \vee \operatorname{sr}\left(M\left(\mathfrak{I}_{\theta}\right)\right) \\
\leq & ([(n+1) / 2]+1) \vee \sup _{t \in(0,1]} \operatorname{sr}\left(C^{b}((0,1]) \otimes \mathfrak{B}_{\theta, t}\right)=[(n+1) / 2]+1, \\
& \operatorname{csr}\left(C_{0}(\mathbf{T} \backslash\{1\}) \otimes \mathfrak{A}_{\theta}\right) \leq \operatorname{csr}\left(C\left(\mathbf{T}^{n+1}\right)\right) \vee \operatorname{csr}\left(\mathfrak{I}_{\theta}\right) \\
\leq & ([n / 2]+1) \vee \sup _{t \in(0,1]}\left(\operatorname{csr}\left(C_{0}((0,1]) \otimes \mathfrak{B}_{\theta, t}\right) \vee \operatorname{sr}\left(C_{0}((0,1]) \otimes \mathfrak{B}_{\theta, t}\right)\right)=[n / 2]+1 .
\end{aligned}
$$

REMARK. The estimate $\operatorname{csr}\left(\Gamma\left(\mathbf{T},\left\{\otimes^{n} L_{\theta}(p, q)\right\}_{\theta \in \mathbf{T}}, \mathfrak{F}\right)\right) \leq \operatorname{csr}\left(C\left(\Pi^{n} L(p, q)\right)\right)$ would be deduced from calculation of the cohomotopy or homotopy groups of the lens spaces (cf. $[\mathrm{Sh}])$.

AcKnowledgment. The author would like to thank Professor K. Matsumoto for some comments about his methods for some noncommutative manifolds, and thank Professor S. Tsukuda for some discussion about elementary topological geometry, and also thank the referee for some suggestions for revision.

\section{References}

[APT] C. A. Akemann, G. K. Pedersen and J. Tomiyama, Multipliers of $C^{*}$-algebras, J. Funct. Anal. 13 (1973), 277-301.

[AP] J. ANDERSON and W. PASCHKE, The rotation algebra, Houston J. Math. 15 (1989), 1-26.

[B1] B. BLACKADAR, K-theory for Operator Algebras, Second Edition, Cambridge (1998).

[BKR] B. BlackAdar, A. KumJiAn and M. RøRDAM, Approximately central matrix units and the structure of noncommutative tori, K-Theory 6 (1992), 267-284.

[BP] L. G. BRown and G. K. PEDERSEN, $C^{*}$-algebras of real rank zero, J. Funct. Anal. 99 (1991), 131-149.

[DNNP] M. DĂDĂRlat, G. NAGY, A. NÉmethi and C. PASNICU, Reduction of toplogical stable rank in inductive limits of $C^{*}$-algebras, Pacific J. Math. 153 (1992), 267-276.

[Dx] J. DixMIER, $C^{*}$-algebras, North-Holland (1962).

[Eh] N. Elhage Hass AN, Rangs stables de certaines extensions, J. London Math. Soc. 52 (1995), 605-624.

[EE] G. A. Elliott and D. E. Evans, The structure of the irrational rotation $C^{*}$-algebra, Ann. Math. 138 (1993), 447-501.

[EL1] G. A. ELLiott and Q. Lin, Cut-down method in the inductive limit decomposition of noncommutative tori, J. London Math. Soc. 54 (1996), 121-134.

[EL2] G. A. ELliotT and Q. LIN, Cut-down method in the inductive limit decomposition of noncommutative tori, II: The degenerate case, Operator Algebras and Their Applications, Fields Ints. Commun. 13 (1997), 91-123.

[F] J. M. G. FELL, The structure of algebras of operator fields, Acta Math. 106 (1961), 233-280.

[Ln] Q. LIN, Cut-down method in the inductive limit decomposition of noncommutative tori, III: A complete answer in 3-dimension, Commun. Math. Phys. 179 (1996), 555-575.

[Mt] K. Matsumoto, Non-commutative three dimensional spheres, Japan. J. Math. 17 (1991), $333-356$.

[MT] K. Matsumoto and J. Tomiyama, Non-commutative lens spaces, J. Math. Soc. Japan 44 (1992) $13-41$.

[Mp] G. J. Murphy, $C^{*}$-algebras and Operator Theory, Academic Press (1990). 
[Ng] K. Nagami, Dimension Theory, Academic Press (1970).

[NOP] M. NAGISA, H. OSAKA and N. C. Phillips, Ranks of algebras of continuous $C^{*}$-algebra valued functions, Canad. J. Math. 53 (2001), 979-1030.

[Ns] V. Nistor, Stable range for tensor products of extensions of $\mathcal{K}$ by $C(X)$, J. Operator Theory 16 (1986), 387-396.

[Rf1] M. A. RIEFFEL, Dimension and stable rank in the K-theory of $C^{*}$-algebras, Proc. London Math. Soc. 46 (1983), 301-333.

[Rf2] M. A. RIEFFEL, The homotopy groups of the unitary groups of non-commutative tori, J. Operator Theory 17 (1987), 237-254.

[Sh] A. J-L. SHEU, A cancellation theorem for projective modules over the group $C^{*}$-algebras of certain nilpotent Lie groups, Canad. J. Math. 39 (1987), 365-427.

[Sd] T. Sudo, Stable rank of $C^{*}$-algebras of continuous fields, Preprint.

[Wl] D. P. Williams, The structure of crossed products by smooth actions, J. Austral. Math. Soc. (Series A)

47 (1989), 226-235.

[Wo] N. E. WegGe-OlSEN, K-theory and $C^{*}$-algebras, Oxford Univ. Press (1993).

Present Address:

Department of Mathematical Sciences, Faculty of Science,

UNIVERSITY OF THE RYUKYUS,

NISHIHARA-CHO, OKINAWA, 903-0213 JAPAN.

e-mail: sudo@math.u-ryukyu.ac.jp 